\title{
Thème 3
}

\section{Conception et calcul des structures et ouvrages en milieux marin et côtier}

\author{
Rapport général
}

par

\author{
P. Monadier \\ Chef du Service technique central des ports maritimes \\ et des voies navigables (Compiègne)
}

\section{Introduction}

1.1 - Malgré le titre ambitieux du thème 3 , je préciserai d'entrée que les cinq communications qu'il regroupe n'ont en aucune façon la prétention de donner une vue d'ensemble sur les problèmes de conception et de calcul des structures et ouvrages en milieux marin et côtier.

Sur le plan de la conception et du calcul tout d'abord, elles se limitent pour l'essentiel à décrire l'évolution des moyens d'essais en laboratoire et à donner quelques indications sur des résultats obtenus récemment en ce qui concerne la propagation de la houle à l'approche du rivage et les digues à talus.

Sur le plan des structures et ouvrages, elles ne s'intéressent explicitement qu'aux digues à talus, en ne mentionnant d'autres types d'ouvrages que par le biais des moyens d'essais.

Sur le plan du milieu, enfin, il ne s'agit pratiquement que du milieu côtier et il n'est pas question des nombreux types d'ouvrages offshore qui sont réalisés.

Cette limitation des sujets a été volontaire, car il nous est apparu plus intéressant et significatif d'examiner, dans un colloque tel que celui-ci, quelques questions assez étroitement sélectionnées plutôt que d'évoquer très superficiellement une vaste gamme de sujets.

1.2. - Je pense que les questions traitées peuvent être regroupées en trois centres d'intérêt que j'aborderai successivement et qui peuvent être désignés comme suit :

- techniques de laboratoire;

- quelques résultats sur la houle;

- stabilité des carapaces de digues à talus.

1.3. - Pour chacun de ces centres d'intérêt, je présenterai tout d'abord très succinctement les communications qui s'y rattachent, laissant aux auteurs le soin d'appeler l'attention sur certains points et de développer plus amplement certaines parties qui leur apparaissent le justifier, puis j'indiquerai les réflexions que m'a inspirée la lecture des textes et je formulerai quelques questions destinées à amorcer les discussions. 


\section{Techniques de laboratoire}

2.1. - Elles font essentiellement l'objet de deux communications :

- celle de MM. Chaloin et Darras, retraçant dix ans d'essais en houle aléatoire au Laboratoire national d'hydraulique:

- celle de M. Moguilny, relative à l'emploi de la houle aléatoire dans les essais de laboratoire et à la technique de production de cette houle au Laboratoire central d'hydraulique de France.

Mais la communication de MM. Rouch et Canel donne également quelques indications sur les méthodes expérimentales utilisées par SOGREAH.

2.1.1. - La communication de MM. Chaloin et Darras donne successivement :

- la description des deux installations à houle aléatoire existantes : grande cuve d'agitation et canal d'étude de l'action des éléments:

- la description des méthodes de mise en œuvre automatisée de ces équipements :

- pilotage automatique et suivi immédiat des essais,

- contrôle de la simulation (génération du signal et analyse du signal incident),

- analyse des mesures de houle (analyse spectrale et analyse vagues par vagues);

- l'exposé de quelques résultats obtenus dans des domaines variés : conception des digues à talus, essais de comportement de structures.

Elle souligne, en conclusion, l'intérêt des modèles physiques faisant appel à la houle aléatoire.

2.1.2. - La communication de M. Moguilny comporte :

- un exposé des principes généraux auquel obéit le système intégré de production de la houle aléatoire du LCHF;

- une description des particularités des solutions adoptées : définition de la houle à reproduire, élaboration du signal d'entrée, vérin hydraulique à commande numérique, mesure de la houle observée dans les bassins (acquisition des données, traitement des données,...);

- une présentation de la méthodologie des applications, avec quelques exemples.

\section{2. - Ces communications m'inspirent trois types de} réflexions :

a) Il m'apparaît tout d'abord indispensable de souligner les progrès très substantiels accomplis depuis une vingtaine d'année en matière de modèles réduits physiques. Ces progrès se traduisent sur différents plans :

- amélioration de la fiabilité des résultats, permettant de se reposer maintenant sans craintes sur les modèles pour concevoir les ouvrages;

- amélioration de la finesse des résultats, permettant d'éviter des simplifications abusives des phénomènes décrits et d'obtenir en conséquence des indications beaucoup plus réalistes que dans le passé;

- réduction des délais d'exécution des essais, en évitant les longues phases préparatoires, les arrêts périodiques et les délais de dépouillement et d'exploitation des résultats;
- réduction du coût des essais, qui les rendent abordables pour des ouvrages même relativement modestes.

Plusieurs éléments ont concouru à ces progrès :

- une meilleure connaissance des lois physiques, permettant une meilleure modélisation (analyse de la houle par exemple);

- une meilleure représentation des phénomènes rendue possible par des améliorations technologiques;

- l'élimination des phénomènes parasites, grâce à l'expérience;

- la mise au point de capteurs précis et fiables;

- l'intervention massive de l'électronique et des calculateurs pour le pilotage des modèles, le contrôle de leur fonctionnement et l'exploitation des résultats.

b) Les modèles réduits physiques et les modèles mathématiques sont assez souvent présentés comme concurrents. Mais cette attitude est en fait fondée sur une vue assez superficielle des problèmes. Je pense qu'en réalité :

- les modèles mathématiques permettent, en matière de houles, de courants, d'interactions avec les ouvrages, d'explorer rapidement, complètement et économiquement, de larges gammes de situations et de possibilités, voire de traiter intégralement certains problèmes relativement simples;

- les modèles physiques permettent seuls, dans bien des problèmes, d'approfondir suffisamment les études, d'aboutir à des résultats suffisamment précis pour assurer une conception correcte des ouvrages, et restent seuls utilisables pour des géométries compliquées, des fonds variables, des phénomènes non linéaires, tels le déferlement, les fortes houles, les effets de frottement visqueux...

De plus les modèles physiques sont indispensables dans bien des cas pour assurer un développement fiable des modèles mathématiques dont ils permettent, conjointement avec des observations et mesures réelles, de vérifier et d'étalonner les méthodes de calcul et les résultats, et dont ils permettent de cerner les limites de validité, ce qui n'est pas toujours possible directement. Il n'est pas exclu toutefois que, dans certains cas, l'utilisation de modèles mathématiques permette de déceler des anomalies sur des modèles physiques.

$\mathrm{Si}$ le développement des matériels informatiques et des codes de calcul doit conduire à augmenter constamment et rapidement la gamme d'études susceptibles d'être traitées par des modèles mathématiques, l'activité des modèles physiques ne me paraît pas pour autant vouée à la régression dans la mesure où l'utilisation des codes de calcul permettra dans bien des cas, soit de dégrossir bien plus valablement qu'auparavant des études qui seront poursuivies sur modèles physiques, soit de traiter complètement des études qui n'auraient sans doute jamais été effectuées auparavant, pour des raisons de coût notamment.

c) L'utilisation des modèles, qu'ils soient physiques ou mathématiques, suppose qu'aient été auparavant recueillies des données naturelles fiables, suffisamment abondantes et adaptées aux problèmes à traiter. Force est de reconnaître que, du moins pour les problèmes pratiques dont je peux avoir régulièrement connaissance, ces conditions ne sont pas pas convenablement remplies. 
Des efforts importants restent à effectuer dans ce domaine, pour :

- d'une part disposer d'appareils de mesure simples, robustes, raisonnablement économiques;

- d'autre part, définir des campagnes de mesures convenablement adaptées aux problèmes à traiter.

2.3. - Je terminerai l'examen de ce premier "sousthème " par quelques questions :

a) Les rôles respectifs des modèles réduits physiques et des modèles mathématiques, que j'ai brièvement esquissés il y a quelques instants, de mon point de vue, peuvent-ils être mieux cernés ? Quelles sont les évolutions marquantes à attendre au cours des cinq prochaines années? Les opinions sont-elles convergentes sur ces questions?

b) N'y a-t-il pas disproportion entre les possibilités actuelles des modèles physiques, et les données naturelles dont on dispose pour les introduire dans les modèles ? Par exemple, n'utilise-t-on pas abusivement des caractéristiques types de houles que l'on possède (spectres de Pierson-Moskowitz ou du Jonswap) sans vérifier si elles sont réellement adaptées au cas à traiter ?

c) L'utilisation de la houle aléatoire est-elle toujours nécessaire pour traiter des cas courants, par exemple en matière d'agitation portuaire ou de digues à talus ? Une des approches de l'ensemble des études effectuées par le $\mathrm{LNH}$ en matière de digues au cours des dernières années semble avoir précisément eu pour objet de limiter les études en houle aléatoire. Les idées sont-elles claires sur les relations d'équivalence à utiliser entre houles aléatoires et houles régulières?

d) Y a-t-il encore beaucoup d'amélioration à attendre de l'automatisation de l'exploitation des modèles physiques, sur le plan des coûts, des délais, de la qualité technique des résultats?

e) Les méthodes de production de la houle aléatoire utilisées par le LNH et SOGREAH sont-elles bien différentes de celles utilisées par le LCHF? SOGREAH semble, elle, recourir au bruit blanc et utiliser le produit de convolution réalisé par un synthétiseur électronique d'un bruit blanc par une réponse impulsionnelle respectant le spectre énergétique de consigne (communication de MM. Rouch et Canel).

Le LNH utilise une méthode dite de phase aléatoire, telle que proposée par Goda.

f) Comment sont engendrés des groupements de vagues dans la technique LCHF de production de la houle aléatoire?

g) Comment sont déterminés expérimentalement les coefficients de réflexion des ouvrages en houle aléatoire ?

L'élimination des réflexions parasites sur modèle est-elle, dans tous les cas, satisfaisante?

\section{Quelques résultats sur la houle}

3.1. - La communication de MM. Rouch et Canel présente des résultats obtenus par SOGREAH sur la limitation des hauteurs des houles aléatoires par la profondeur, lors d'essais en canal et d'essais en cuve exécutés respectivement pour l'étude d'une digue à talus et l'étude d'une prise d'eau.
Ces résultats devant être présentés plus finement par les auteurs, je me borne à indiquer très brièvement qu'ils ont constaté :

- une modification de l'histogramme des hauteurs (à la limite du déferlement) par rapport à la répartition classique de Rayleigh, avec accroissement de la population de lames de faibles hauteurs;

- des valeurs des rapports $H_{d} / \mathrm{d}, H_{1} / d, H_{m q} / d$ peu variables avec la période de pic du spectre d'énergie.

Ces résultats sont en bonne concordance avec ceux obtenus par Seelig dans des conditions comparables.

3.2. - Je formulerai quatre remarques sur ces résultats :

- Ils sont incontestablement intéressants car ils permettent de dépasser, dans un domaine d'intérêt pratique incontestable, celui des ouvrages étudiés dans les conditions du déferlement, la formule simpliste généralement utilisée, soit $H b / d b=0,78$.

- Ils n'ont été obtenus qu'avec des pentes de fonds très faibles ( $1 \%$ et $1,7 \%)$.

- On constate que $H_{\max }$ est limité à $1,8 H_{m i q}$, ce qui donne en moyenne $H_{\max } / d=0,77$, soit précisément la relation rappelée ci-dessus.

- La planche 3 ne laisse pas entrevoir que, pour $d / g T^{2} p$ très faible, $\mathrm{Ho} / \mathrm{d}$ puisse être de l'ordre de 1 .

3.3. - Ces essais et ces résultats me paraissent appeler quelques questions :

- Comment est déterminée dans de tels essais la limite du déferlement?

- Oủ est, précisément, mesurée la profondeur $d$ prise en compte dans les rapports ?

- L'explication donnée sur l'absence de différence notable entre les mesures effectuées en présence d'un ouvrage réfléchissant et d'un ouvrage peu réfléchissant peut-elle être développée?

\section{Stabilité des carapaces de digues à talus}

4.1. - Cette question fait essentiellement l'objet de deux communications :

- celle de M. Barailler sur le choix de l'amplitude à employer, dans l'utilisation de la formule de Hudson, pour la conception des digues à talus,

- celle de MM. Carpentier et Brosselard - Faidherbe présentant les résultats de leurs travaux relatifs à l'influence de la période de la houle sur la stabilité des digues à talus,

mais elle est également abordée dans la dernière partie de la communication de MM. Chaloin et Darras.

4.1.1. - Je voudrais tout d'abord situer ces communications dans les préoccupations actuelles relatives aux digues à talus, qui sont essentiellement de trois ordres :

- stabilité hydraulique, concernant le comportement sous l'action de la houle des carapaces supposées constituées de blocs infiniment résistants placés sur des souscouches et un noyau supposés sans risque d'instabilité; - stabilité mécanique, concernant la rupture par la houle 
des blocs de carapace eux-mêmes, à la suite des oscillations et des chocs auxquels ils sont soumis;

- stabilité au glissement d'ensemble, dont la réduction par suite du développement de pressions interstitielles importantes à l'intérieur de l'ouvrage pourrait, selon certains auteurs, être à l'origine de la destruction de quelques digues.

Aucune communication ne porte sur le troisième type de préoccupation pour lequel j'indiquerai simplement que certaines études sont en cours, notamment sur la digue de Mohammedia, au Maroc, et sur la nouvelle digue Est du port de Calais. Elles comportent d'une part des campagnes de mesures de pressions interstitielles, à l'intérieur de l'ouvrage, d'autre part des essais d'interprétations théoriques.

4.1.2. - La communication de M. Barailler porte sur les deux premiers types de questions et se propose de donner des indications pratiques pour le prédimensionnement des digues à talus, sous la forme de deux recommandations pour l'utilisation de la formule de Hudson :

- prise en compte de la hauteur significative comme valeur caractéristique de la houle,

- réduction par un facteur de l'ordre de 2 du coefficient $K_{D}$ de cette même formule pour se prémunir contre les risques de rupture des blocs.

4.1.3. - La communication de MM. Carpentier et Brosselard-Faidherbe porte en fait sur deux questions distinctes intéressant la stabilité hydraulique.

a) Dans une première partie, ils exposent les résultats des recherches bibliographiques et des essais en canal qu'ils ont effectués pour analyser l'influence de la période de la houle sur la stabilité globale d'une carapace. Ces travaux, dont le caractère partiel est souligné, leur permettent néanmoins de formuler des conclusions très intéressantes que je me borne à mentionner brièvement en leur laissant le soin de les développer :

- L'agressivité des houles se manifeste pour une large gamme de périodes, sur laquelle le coefficient de stabilité de Hudson varie de 1 à 3. Dans les conditions d'essais :

- avec les périodes de 10 à $12 \mathrm{~s}$, la ruine de l'ouvrage est apparue le plus rapidement, par perte de blocs ou de groupes de blocs;

- avec la période de $20 \mathrm{~s}$, la ruine est intervenue par fluidisation de la carapace.

- L'influence de la période paraît essentiellement s'exercer par la détermination du type de déferlement, le déferlement par effondrement s'avérant le plus redoutable. - Plus que la période, ce sont en fait les conditions de déferlement sur les digues, conditionnées par la profondeur et la pente des fonds, et la durée des tempêtes qui apparaissent les facteurs les plus importants.

b) Dans une seconde partie, les auteurs examinent, pour le même type de carapace que dans les essais précédents, les efforts appliqués à un groupe de blocs et les variations du niveau de l'eau de part et d'autre de la carapace.

Pour la position choisie des blocs, il est apparu que les risques d'instabilité apparaissent lors du retrait de la lame, et l'existence d'une période critique a été mise en évidence, pour laquelle ces risques sont les plus élevés.

\section{2.}

4.2.1. - Je ferai pour ma part trois observations sur les conclusions de M. Barailler :

a) Le fait de prendre $H_{s}$ pour la stabilité hydraulique me paraît plus hardi que ce qui est maintenant assez couramment admis, me semble-t-il. Les recommandations du CERC préconisent, comme le $\mathrm{LNH}$, la prise en compte de $H_{1 / 10}$. En outre, les essais effectués au LNH qui sont mentionnés :

- n'ont pas tous pris en compte des pentes des fonds de $10 \%$ susceptibles effectivement d'infléchir notablement les résultats;

- n'aboutissent à préconiser $H_{1 / 20}$ que pour des durées de tempêtes de l'ordre de $6 \mathrm{~h}$, la valeur de l'ordre de $H_{1 / 10}$ correspondant à des durées de tempêtes de l'ordre de $3 \mathrm{~h}$, et s'efforcent de donner des indications quantitatives sur l'accroissement du taux de dommages en fonction de la durée de la tempête.

b) La réduction de $K_{D}$ préconisée pour tenir compte des risques de rupture des blocs constitue une approche assez radicale, du traitement de cette question difficile pour le projeteur.

c) La conclusion pratique, qui consiste en une division par 2 de $K_{D}$ pour tenir compte des risques de rupture et une prise en compte de $H_{s}$ au regard des problèmes hydrauliques, revient à maintenir $K_{D}$ et retenir $H_{1 / 10}$, puisqu'on aboutit dans les deux cas, à densité constante du matériau, à un doublement du poids des blocs par rapport aux usages plus anciens qui consistaient à prendre en compte $H$, sans tenir compte des risque de rupture.

4.2.2. - La communication de MM. Carpentier et Brosselard-Faidherbe me suggère, quant à elle, deux remarques.

a) Les résultats globaux présentés sont encore très partiels et leur validité ne peut pas, sans essais complémentaires, être étendue bien au-delà des conditions expérimentales pour lesquelles ils ont été obtenus.

Ils sont néanmoins fort intéressants car :

- la mise en évidence d'une plage de périodes d'agressivité, avec deux zones de périodes particulièrement défavorables (déferlement par effondrement et fluidisation des carapaces) peut être un guide précieux pour des essais pratiques concernant des cas particuliers;

- la constatation du caractère conservatif, mais sans excès abusifs, de la formule de Hudson constitue une vérification très utile de son intérêt.

b) Les résultats relatifs aux efforts sur les blocs apportent un éclairage précieux sur le mode d'attaque des carapaces, mais ne peuvent encore avoir, eux aussi, qu'une signification très partielle.

4.3. - Je terminerai, une dernière fois, par quelques questions.

a) Comment ont été effectués les essais de SOGREAH tendant à confirmer la validité d'une prise en compte de $H_{s}$ en ce qui concerne la stabilité hydraulique (déferlement possible, durée des tempêtes,...) ?

b) L'auteur pourrait-il apporter quelques précisions sur les considérations qui l'amènent à proposer une division 
de $K_{D}$ par deux pour tenir compte des risques de rupture des blocs? Il semble en tout état de cause que la réduction appliquée devrait être assez différente selon les types de blocs, et en particulier la fragilité plus ou moins importante et les relations mécaniques plus ou moins étroites avec les voisins résultant de leur forme même.

c) MM. Carpentier et Brosselard-Faidherbe pourraient-ils donner quelques indications sur les possibilités et les limites d'extension des résultats globaux qu'ils ont obtenus en ce qui concerne l'influence de la période de la houle sur la stabilité d'une carapace?

d) Les relations entre les mouvements d'eau dans la carapace (positions et vitesses) et les efforts sur les blocs ne pourraient-elles pas faire l'objet d'une tentative d'approche quantitative?

e) Ne serait-il pas souhaitable d'engager, du moins sur le plan national, une certaine normalisation, ou codification, des essais relatifs aux digues (préparation des modèles, houles de tassement, cycles d'essais, définition du taux de dommages, mesure des franchissements,...). Bien que difficile, une telle procédure ne me paraît pas a priori à exclure et des améliorations ont déjà été obtenues dans des secteurs où les difficultés étaient également importantes tels les essais de chargement de pieux.

Elle me semble une condition nécessaire pour que les résultats obtenus dans différents laboratoires puissent être convenablement comparés.

\section{Conclusion}

J'espère simplement vous avoir donné le regret de ne pas avoir tous lu à fond les textes des communications présentées, et vous avoir donné l'envie, d'une part d'entendre de la part des auteurs des indications plus précises que celles que j'ai rapidement données sur leurs communications, d'autre part, de leur poser bon nombre de questions. 